\title{
Complutum
}

ISSN: 1131-6993

\section{Innovando (desde) el ecosistema arqueológico profesional: hacia la cohesión del colectivo arqueológico}

\author{
Carlos Caballero¹, Lourdes López Martínez, José Manuel Illán, Ernesto Agustí y Marta Cuesta
}

Recibido: 26/04/2021 / Aceptado: 09/08/2021

Resumen. Más de treinta años después de la cesión a las Autonomías de las competencias arqueológicas, la arqueología profesional se enfrenta a un panorama complejo: el colectivo, adaptado a un mercado cambiante, no ha resuelto la falta de cohesión de la profesión, la disparidad de criterios de las Administraciones y la falta de relación con una ciudadanía cuyo patrimonio es el objeto de estudio de la profesión, pero a la que no se transmite la importancia y la necesidad de su estudio. Tras dos reuniones celebradas en Cádiz (2018) y Madrid (2019), surgió el proyecto Innovando (desde) el Ecosistema Arqueológico Profesional, que fue posible gracias a una ayuda de la Dirección General de Industrias Culturales y Cooperación del Ministerio de Cultura y Deporte, y consistió en la celebración de tres reuniones entre noviembre de 2019 y diciembre de 2020. El proyecto permitió trabajar juntos a profesionales de todo el Estado y, como resultado, se constituyó la Plataforma de Profesionales de la Arqueología (PEPA), para aglutinar a los profesionales que formen parte de ella, superar los marcos territoriales de los colegios y asociaciones, dignificar la profesión y encontrar formas adecuadas de diálogo entre quienes trabajan en el patrimonio arqueológico y la ciudadanía.

Palabras Clave: Arqueología Profesional, Asociacionismo, Colegios profesionales, Patrimonio arqueológico, Arqueología, Sociedad

\section{[en] Innovating (from) the professional archaeological Ecosystem: Towards the cohesion of the archaeological collective}

\begin{abstract}
Over thirty years after the transfer to the Autonomous Communities of the competences in archaeology, professional archaeology faces a complex panorama: the professional collective has adapted to a changing market, but without resolving the lack of cohesion of the profession, the disparity of criteria of the Administrations and the lack of relation with a citizenship whose heritage is the object of study of the profession, but to which the importance and necessity of its study is not transmitted. After two meetings held in Cadiz (2018) and Madrid (2019), a project called Innovating (from) the Professional Archaeological Ecosystem, which was made possible thanks to the assistance of the Dirección General de Industrias Culturales (Ministry of Culture and Sports), and consisted of three meetings between November 2019 and December 2020. The project allowed professionals from all over the State to work together and, as a result, the Platform of Archaeology Professionals (PEPA) was established to rally all the professionals taking part in it, to go beyond the territorial frameworks of colleges and associations, dignify the profession and find appropriate forms of dialogue between professionals working with the archaeological heritage and the public, to which it belongs. Keywords: Professional Archaeology, Associationism, Archaeological Heritage, Professional Colleges, Archaeology, Society
\end{abstract}

Sumario. 1. Introducción: la necesidad de (re)ubicar una profesión. 2. Antecedentes: reflexiones anteriores. 3. El Proyecto Innovando... un paso hacia la cohesión. 4. Caminando hacia el futuro: la consolidación del colectivo. Bibliografía.

Como citar: Caballero, C. et al. (2021): Innovando (desde) el ecosistema arqueológico profesional: hacia la cohesión del colectivo arqueológico. Complutum, 32(2): 245-259.

1 Plataforma de Profesionales de la Arqueología, "PEPA" (info@plataformadearqueologia.es) 


\section{Introducción: la necesidad de (re)ubicar una profesión}

Hace apenas unos días, una de las personas que han redactado conjuntamente este texto tuvo que contestar, para la prensa, a una pregunta recurrente: "¿para qué sirve un arqueólogo en una obra?". A una pregunta similar tenemos que enfrentarnos los profesionales de la arqueología, algo así como "exactamente, tu trabajo, ¿en qué consiste?". La pregunta puede venir formulada por cualquiera de las personas que trabajan en la obra, sea cual sea su oficio o su cometido en la construcción, convirtiendo, probablemente, a la arqueología en la única profesión relacionada con la construcción cuyo cometido desconoce la mayoría de las personas implicadas en una obra. A veces, incluso, es el promotor, a menudo quien finalmente contrata al equipo arqueológico, el que ignora qué papel desempeñan esos profesionales a los que ha contratado simplemente "porque para darme la licencia me exigen un arqueólogo".

No deja de ser sorprendente que, casi cuatro décadas después de la promulgación de la Ley 16/85, del Patrimonio Histórico Español, que dio carta de naturaleza a la generalización de las intervenciones arqueológicas en España, la arqueología siga siendo una pro- fesión desconocida. Desconocida en sus métodos, en sus objetivos y en sus fines. Y, por supuesto, una profesión desregulada, sin reconocimiento como tal por parte de la normativa sectorial vigente (la mencionada Ley del Patrimonio Histórico Español, las correspondientes legislaciones autonómicas y las normas medioambientales autonómicas que también influyen en el trabajo arqueológico) y de la que la sociedad no tiene sino una idea muy alejada de la verdadera realidad. Ese es el punto de partida de la iniciativa que se resume en estas páginas.

\section{Antecedentes: reflexiones anteriores}

El planteamiento del problema, la búsqueda de sus raíces, no es nuevo: es algo que se lleva haciendo en la profesión desde prácticamente su puesta en marcha, a mediados de los años 80 del siglo XX. Ha habido muchas reflexiones publicadas en diversos ámbitos, compartidas en foros variados, pero quizás la que mejor resume la historia de la arqueología profesional en España sea esta, publicada en 2018: "La arqueología en España está en crisis. Para nosotros nunca ha dejado de estar en crisis. Nació en crisis, creció en crisis y ahora atraviesa su particular crisis de los 40" (López Martínez y Martín Alonso 2018: 225).

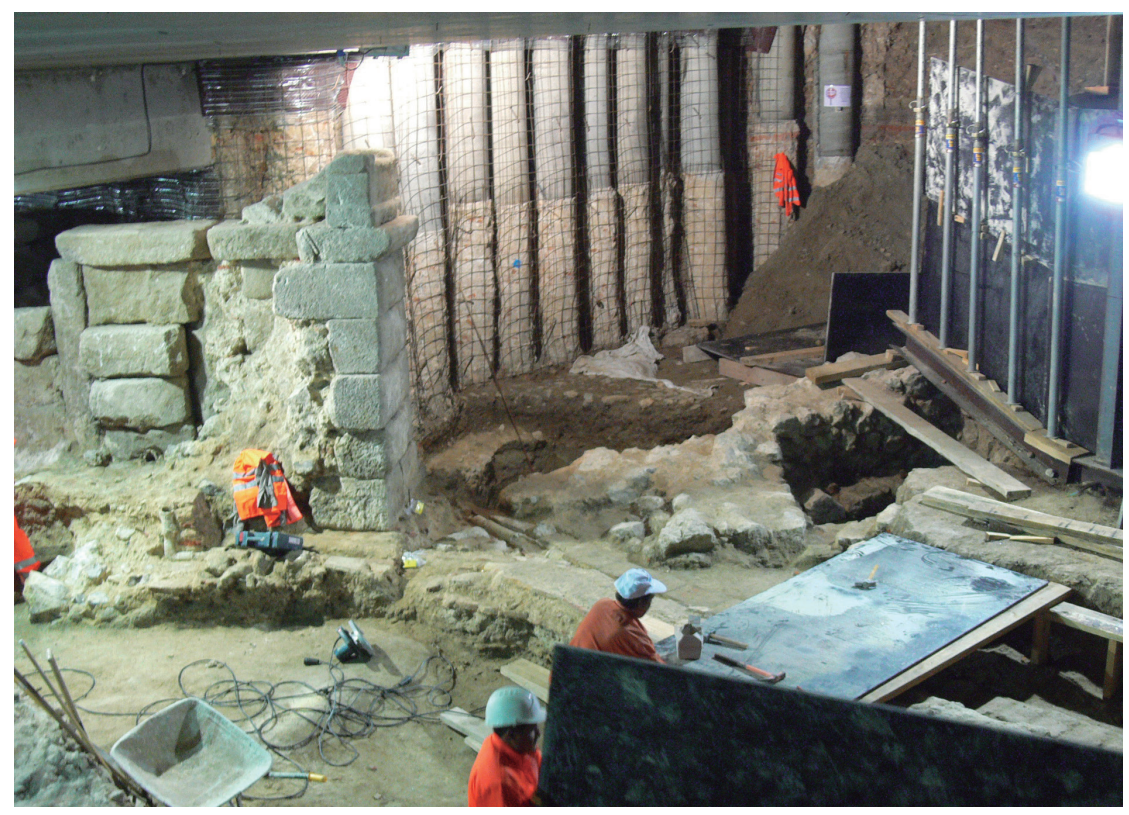

Figura 1. El trabajo arqueológico en una obra civil: la estación de Metro de Ópera, en Madrid (C. Caballero, 2010). 
Probablemente no sea este el lugar, porque el espacio se nos iría sin abordar el asunto central de este texto, para resumir las múltiples visiones que se han publicado en los últimos treinta años acerca de la arqueología profesional en España, pero sí creemos prudente reseñarlas brevemente aquí para que quienes lean este trabajo puedan acudir a ellas y contrastar las diferentes opiniones que componen este amplio mosaico.

El problema de partida, probablemente, estuviera ya en los primeros pasos dados por la profesión, al amparo de la Ley 16/85 del Patrimonio Histórico Español, que supuso la transferencia, desde el Estado a las comunidades autónomas, de las competencias referentes a la gestión del patrimonio arqueológico (Mena 1991). Esa cesión de competencias significó, por una parte, la creación de cuerpos técnicos en las distintas Administraciones autonómicas, con sus diferentes relaciones entre Administraciones locales, analizadas por Alicia Castillo (2004) y, por otra, el comienzo de la actividad profesional de decenas de arqueólogos y arqueólogas que hasta el momento ejercían su trabajo fuera de cualquier regulación. Fue así cómo, desde los primeros momentos se puso de manifiesto que la profesión iba a enfrentarse a dos problemas endémicos: la desregulación y la indefinición. Sobre esta última, todavía en los últimos años del siglo XX se seguían publicando, en escritos dirigidos a un público amplio, los diferentes tipos de intervenciones arqueológicas (Fernández Ugalde et al. 1998: 12). La naturaleza misma de las diferentes intervenciones, en función de su promotor y de los objetivos, ha sido siempre objeto de debate, y aun en 2010 había quien separaba las intervenciones "de urgencia" de las "de investigación" (así, en Moya 2010: 11).

No solo la desigual aplicación de esa Ley estatal, en su trasposición a las diferentes comunidades, se tradujo en actitudes, comportamientos y normativas distintas en la gestión del patrimonio común, sino que la Ley tuvo, además, un efecto indeseado y que, probablemente, el legislador no había calculado: al socializar la financiación de los estudios arqueológicos, implicando al promotor de obras en zonas arqueológicas mediante el procedimiento de que fuera él quien financiase la investigación arqueológica, se obtuvo el efecto contrario del que se perseguía (que era extender la idea de que el patrimonio ar- queológico es de toda la sociedad y todos debemos comprometernos en su cuidado), haciendo que las intervenciones arqueológicas se adjudiquen, en simple subasta, al mejor postor, al precio más barato, independientemente de que la persona autora de la oferta tenga competencia suficiente. Eso es algo que, en último extremo, debería regular la Administración, pero en la inmensa mayoría de los casos se conceden autorizaciones sin entrar a valorar la calidad de la propuesta presentada o si el curriculum de los solicitantes es suficiente. La adjudicación de intervenciones arqueológicas "al mejor postor" es una queja recurrente en la literatura al uso (Baena 2007; Gómez Hernanz 2007 o Ruiz Taboada 2014, entre otros).

Se ha debatido mucho también sobre la labor de las Administraciones, las Universidades y las Instituciones, en particular sobre su influencia en la labor de la arqueología profesional. En ese sentido, habría que destacar reflexiones como las de Sergi Selma quien, al hablar de la gestión del Servicio de Investigación Arqueológica y Prehistórica de la Diputación de Castellón, señala que "La viabilidad de algunos servicios, y más en el ámbito del patrimonio cultural, no puede cuantificarse sólo en términos económicos. Y eso es algo que el colectivo viene reivindicando desde siempre, y no podemos darle la espalda ahora que las cosas no vienen de cara. Las reivindicaciones del colectivo arqueológico deberían continuar por reclamar inversiones en patrimonio, en tanto en cuanto funcionen como punta de lanza para el mantenimiento de una cierta actividad profesional en nuestro sector y, sobre todo, en otros sectores productivos de la sociedad" (Barrachina y Selma 2014). Muchas voces aplauden el esfuerzo realizado por la Administración incrementando sus plantillas y sus inversiones, pero coinciden también en que ha de ser más ágil y no permitir, con su ambigüedad y sus arbitrariedades, que se abra hueco a la mala praxis profesional (Polo 2007: 132). Javier Baena (2007: 118), aun reconociendo, como suele ser norma en la arqueología profesional, el enorme esfuerzo hecho por la Administración desde 1985, señala también que la existencia de asimetrías y disimetrías entre los distintos ámbitos de la Administración, la falta de recursos humanos o económicos, e incluso cuestiones de índole personal plantean a la Administración serios impedimen- 
tos a la hora de hacer efectivo un diseño que, al menos a nivel teórico, se presenta como infalible.

Se pide, en fin, a la Administración que ejerza de contrapeso en las relaciones que, entre el arqueólogo y el promotor, se generan en las obras: "Entre la presión que reciben los profesionales de la arqueología en una obra, destaca la del promotor, normalmente el menos interesado en el aspecto científico del trabajo arqueológico: en esas circunstancias, cuando la presión se traduce en pedir al arqueólogo que realice su trabajo rápido y acogiéndose a unos mínimos, debe ser la Administración la que garantice la calidad del trabajo arqueológico" (Gómez Hernanz, 2007), pero la Administración no suele implicarse en esa labor reguladora.

También se ha destacado la nula o escasa relación entre los profesionales que practican la arqueología desde sus diversos ámbitos; en este sentido, el resumen más lúcido es, probablemente, el que hizo José Polo (2007: 131) tras una mesa redonda sobre la situación del sector: "Si excluimos las relaciones personales, el término que mejor expresa la interacción entre los diferentes sectores de la arqueología es la mutua ignorancia", quizá menos exacto quince años después, pero aún en vigor. Algunos autores han abogado por la creación de una sólida red basada en el asociacionismo ya desde la Universidad, enfrentándose al neoliberalismo imperante (en especial, Palomero 2019). En el mismo sentido, se alzan voces frente a la precariedad impuesta por el sistema imperante, como Vigil - Escalera (2011) o, especialmente, Aparicio (2018), que aboga, directamente, por el asociacionismo entre iguales, como en el caso de la Asociación de Dibujantes de Arqueología (ADARQ), ofreciendo como modelo de relación para un colectivo amplio el que ya ha demostrado su eficacia en uno más específico.

Entre las recopilaciones más recientes de contrastes de pareceres es imprescindible incluir aquí los diferentes trabajos recopilatorios coordinados por Jaime Almansa, una de las voces críticas más activas actualmente en el colectivo, y al que le acompaña una visión multifacética derivada de su experiencia profesional. En esencia, cabría citar dos obras concretas, la dedicada al futuro de la arqueología en España (Almansa 2011), y la que dio carta de naturaleza a la arqueología pública española (Almansa 2013). Entre las muchas opiniones recopiladas, imposibles de resumir aquí, cabe reproducir la idea general de que la arqueología profesional solo puede entenderse como colectivo desde sus diferentes vertientes. En su último trabajo publicado, hasta el momento, Almansa, en el marco del desarrollo del proyecto \#pubArchMED, ofrece una visión amplia de las distintas formas de practicar la arqueología profesional en toda la cuenca mediterránea (Almansa 2021), sin olvidar que muchos de estos planteamientos parten de la tesis doctoral del autor (Almansa 2018).

Diversas voces han insistido también en el objetivo no alcanzado de la difusión del resultado del trabajo arqueológico, algo que algún autor ha llegado a definir como "una cierta incapacidad por parte de los arqueólogos para difundir los resultados de nuestro trabajo (consciente o inconsciente; militante o real)" (Vaquerizo 2015). Hay quien piensa que se valora la necesidad de divulgar y abrir la disciplina al público, pero es algo que no llega a hacerse (Almansa 2021: 286). De la revisión bibliográfica se extrae también la impresión de un pesimismo dominante: "La mayoría de las personas encuentra dificultad a la hora de resaltar aspectos positivos concretos sobre la gestión, aunque algunos de ellos hayan salido a lo largo de la entrevista. Eso sí, se valora de forma muy positiva a los profesionales, aunque con críticas a las viejas generaciones. El empleo es un problema estructural de la arqueología" (Almansa 2021: 286).

Frente a la cierta alergia de buena parte de los miembros del colectivo arqueológico profesional para difundir los resultados de su trabajo más allá de los foros meramente científi$\cos$ (y, a menudo, ni siquiera en ellos), surgen iniciativas que permiten repensar la relación entre arqueología y sociedad, no solo como una presentación de resultados en un lenguaje que se entienda por la mayor parte de la población, sino encaminadas a que la ciudadanía interactúe con su patrimonio arqueológico y con quienes lo recuperan para ella, de modo que el trabajo de estos últimos pueda resultar, en este sentido, lo más eficaz posible. Ha habido diversas iniciativas, especialmente en el segundo decenio de este siglo pero, quizás por sus resultados y por su eficacia en el acercamiento a la ciudadanía, habría que destacar las llevadas a cabo en Alcalá de Henares, 
dentro del proyecto denominado "Alcalá Past Comun" (Corpas et al. 2019), las experiencias llevadas a cabo en Barcelona, tanto por el Grup d'Arqueologia Pública i Patrimoni (Ruiz Martínez y Pastor, 2015), como en el marco del proyecto "Barcelona, una historia de hace 6.000 años" (Gibaja et al. 2018), o la iniciativa llevada adelante en Granada por la Sección de Arqueología del Colegio Profesional de Granada, Almería y Jaén, resumida con el sugerente título de "La Granada invisible" (Mancilla et al. 2018). Finalmente, es muy destacable el proyecto "Cooltoure", desarrollado en Cantabria, y en el que "Gracias al Patrimonio Cultural, impulsamos un modelo de desarrollo turístico sostenible, que implica la participación e interactividad del viajero, promoviendo la inclusión socioeconómica y la generación de oportunidades a la población local, priorizando la conservación y difusión del Patrimonio Cultural sobre su explotación turística, para garantizar su sostenibilidad y evitar su desaparición. Se persigue, además de una alternativa profesional propia, un Territorio Cultural que sepa adaptarse para no desaparecer. Se busca la viabilidad con una alianza y comercialización estratégicas entre Patrimonio Cultural, Turismo y Desarrollo Local" (Astorqui y Díaz 2018). Estos mismos autores construyen una clave de bóveda para el tratamiento de la arqueología del futuro, también para la gestión del patrimonio cultural: "Para nosotros es fundamental trabajar el Territorio pero sobre todo con quien lo habita, con las mujeres y hombres que han creado el paisaje cultural, el patrimonio cultural y las formas de vida, al menos desde el neolítico. Si no somos capaces de trabajar con ellos y para ellos, seguramente perdamos el origen de nuestra razón de ser como sector" (Astorqui y Díaz 2018: 418). Cabría citar aquí también los trabajos de Pastor y Ruiz Martínez (2016), o la tesis doctoral de la propia Ana Pastor (2019), además del trabajo colectivo encabezado por Eva Parga (Parga et al. 2020).

La relación con la sociedad se percibe como fundamental, aunque no siempre se acierte con el método adecuado, incluso se llega a considerar como "medida activa de conservación preventiva" (Vaquerizo 2015). Volviendo al comienzo de este texto, "para conseguir ese respaldo [el de la sociedad] proponemos un activismo arqueológico. Un activismo que lleve la arqueología a la calle. Una arqueología activista no para mejorar sus condiciones propias (que llegará más tarde) ni para aleccionar a nadie, sino para generar debate y reflexión. Partimos de la base de que no hay una conciencia ciudadana de lo que está pasando. Creemos que la mayor parte de la sociedad es "ignorante" de la situación que sufre el patrimonio arqueológico en España. $Y$ es nuestro deber ponérselo delante de forma contundente y clara" (López Martínez y Martín Alonso 2018).

En medio de todas estas casuísticas diversas se sitúan los colegios profesionales, antaño aglutinantes, en torno a sus "secciones de arqueología", de una profesión naciente y hoy con un papel disminuido por diversas circunstancias: la ausencia de la obligatoriedad de la colegiación para ejercer la profesión o la imposibilidad de fijar, para la actividad profesional, honorarios mínimos $\mathrm{o}$, al menos, orientativos, hace que su papel como institución reguladora se haya diluido. Sin embargo, frente a esta realidad sorprende encontrar, aun en 2018, un discurso triunfalista, que desprende la sensación de que, desde los colegios profesionales, casi todo está resuelto en arqueología, y lo que no está aún resuelto, está ya próximo a resolverse:

"El Congreso Nacional de Arqueología Profesional (CNaP 2017) que se ha celebrado los días 4, 5 y 6 de abril del 2017 nace en el seno del Consejo General de Colegios Oficiales de Doctores y Licenciados en Filosofía y Letras y en Ciencias, contando con el apoyo del Colegio Oficial de Doctores y Licenciados en Filosofía y Letras y en Ciencias de Aragón. Emerge con el objetivo de mostrar a la sociedad la calidad de los trabajos que desarrollan los profesionales de la arqueológica y su voluntad de comunicar a la sociedad el resultado de sus investigaciones. El Consejo General de CDL agrupa a más de 1.500 arqueólogos profesionales, de todo el Estado español, que pese a la dura crisis que padecemos han manteniendo los estándares de calidad en su actividad profesional. El principal fundamento del Congreso es incrementar la cultura científica, tecnológica e innovadora en el ámbito de la Arqueología, la presentación de la realidad actual de la arqueología profesional y su actual problemática, las novedades en el campo de la investigación arqueológica profesional en España, la aplicación de nuevas tecnologías en este campo, su plasmación en la didáctica del patrimonio arqueológico, en la musealización 
y la divulgación de yacimientos" (Lorenzo, 2018: 9).

Este discurso, que pese a formar parte del prólogo de las actas de un congreso "de Arqueología Profesional" no se enfrenta a ninguno de los problemas de la profesión, por la sencilla razón de que no los reconoce, y que aboga por un mantenimiento ad infinitum de un status quo cuya eficacia nunca ha sido probada, contribuye decididamente a la esclerotización del sistema y a la puesta en duda del papel de los colegios profesionales.

Frente a la esclerosis de la burocracia centenaria, nuevas formas de asociacionismo se abren paso. Se volverá sobre ello más adelante en este texto, pero son destacables las experiencias de ADARQ, Asociación Nacional de Dibujantes e Ilustradores de Arqueología (Aparicio, 2018), de la Associació d'Arqueolègs de Catalunya (AD'AC) o de ACRE, la Asociación Profesional de Conservadores y Restauradores de España. Nuevas formas de cohesión que nacen, en fin, al amparo de la necesidad de resolver realmente los problemas de una profesión.

La socióloga Eva Parga Dans es bien conocida en el mundo de la arqueología profesional por haber realizado una radiografía del sector basándose en numerosas entrevistas. Entre sus reflexiones, especialmente en las realizadas con posterioridad a 2008, cabe destacar estas, publicadas en la encomiable revista "La Linde" (Parga 2014: 13): "En tan sólo cuatro años la crisis se ha llevado por delante al $42 \%$ de las empresas existentes en 2009 y se ha reducido drásticamente el personal de las empresas que han sobrevivido, manteniendo sólo el 34\% de los puestos de trabajo".

La propia Eva Parga, desde las afueras de la arqueología, apuntaba que aquel contexto derivado de la descomunal crisis de 2008 "representa una oportunidad para sectores como el arqueológico y patrimonial, que tienen el reto de estructurar una apuesta atractiva para involucrar a la sociedad en sus procesos, una sociedad que demanda cultura y no sólo en términos económicos sino como fuente de valor social" (Parga 2014: 15).

Volviendo a los estudios de Eva Parga, "La actividad arqueológica tiene que reinventarse y las posibilidades son muy variadas; a través de preguntas anteriores se apuntaron diferentes oportunidades relacionadas con las actividades culturales y creativas, con el aumento de la demanda cultural por parte de la sociedad y con la especialización y formalización del saber-hacer, y aquí el abanico de posibilidades es muy amplio. El reto está en escuchar a la comunidad, al público y a las instituciones en su sentido más amplio, interpretar el contexto cambiante y ser capaces de adaptar, trabajar y aportar" (Parga 2014: 17).

De todas las reflexiones resumidas aquí, quizás la más ilustrativa, sobre la función del patrimonio cultural y de quienes lo estudian, se deba a Tono Vizcaíno: "No obstante, la

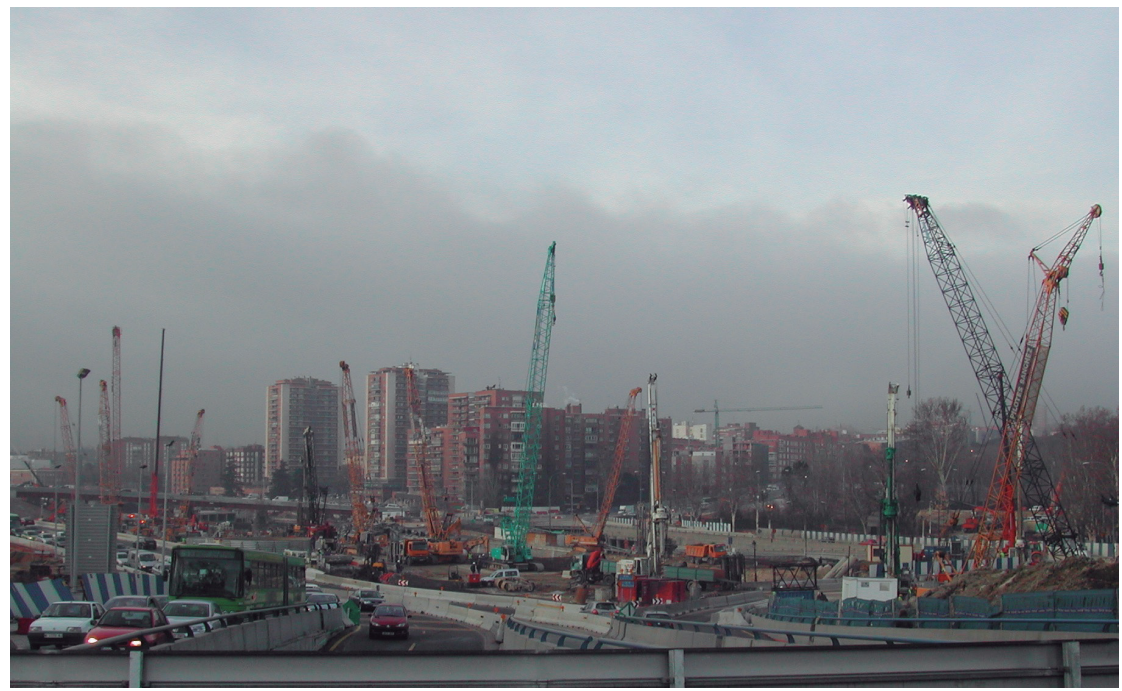

Figura 2. La fugaz coyuntura de las grúas y su impacto en la arqueología: remodelación de la M-30, en Madrid, en el entorno de la ermita de la Virgen del Puerto (C. Caballero, 2006) 
capacidad del patrimonio de asumir lecturas diferenciadas rara vez se traduce en una verdadera pluralidad, pues los intereses de los agentes implicados muchas veces colisionan y no todos tienen las mismas capacidades de intervención y apropiación física y simbólica, de modo que el patrimonio acaba convirtiéndose en un claro reflejo de poder, conflicto y dominación; dado que el patrimonio encarna valores de tipo simbólico, político y económico, tenemos la capacidad de influir a través de él y con nuestro trabajo en la vida de las personas, y no solo desde un punto de vista cultural y económico, que son las vertientes más frecuentemente consideradas al referirnos a la gestión patrimonial, sino también social y político" (Vizcaíno 2015: 194). En definitiva, "urge dejar con mayor frecuencia los laboratorios y las excavaciones y "salir a la calle", pues aunque podamos presuponer muchas cosas, es el contacto con la gente el que realmente nos ayuda a ser conscientes de la situación en que se encuentra nuestra disciplina" (Vizcaíno 2015: 211).

El proyecto que aquí se resume y las consecuencias que de él puedan derivarse son, sin duda, resultado de todas esas visiones, enfrentadas, compartidas o no que, procedentes desde los diferentes ámbitos de la arqueología profesional, han encontrado en una circunstancia común un lugar de encuentro: uno de los principales problemas de la arqueología profesional española es la absoluta dispersión de los profesionales y la nula cohesión que, de ello, se deriva para el colectivo profesional.

\section{El Proyecto Innovando... un paso hacia la cohesión}

Con el amplio bagaje resultante de todas las reflexiones anteriores, la constatada inoperancia de algunas instituciones que deberían tener por fin primordial regular profesionalmente el sector y de resultas, en fin, de la frustrante experiencia que, en lo tocante a ordenación de la profesión, había sido el Congreso Nacional de Arqueología Profesional, al final no más que un trasunto de los antiguos "Congresos Nacionales de Arqueología" (pero con el epíteto adicional de "Profesional"), surgió, como un cambio de rumbo, el Encuentro Estatal de Arqueología Profesional, que organizó el Colegio de Cádiz en noviembre de 2018, y en el que la mayoría de los participantes estaban inscritos en los diferentes colegios profesionales del conjunto del Estado.

Ese primer encuentro no se orientó a la presentación de resultados de diferentes intervenciones arqueológicas, sino que consiguió reunir a profesionales procedentes de todo el Estado para sentarse a debatir, durante tres días, sobre diversos problemas específicos de la profesión, su organización, la interrelación entre profesionales, la conexión con la sociedad o el expolio, entre otros. La declaración final del Encuentro, leída el 18 de noviembre de 2018 en el escenario del Teatro de Títeres de la Tía Norica, emplazado sobre los vestigios visitables del Gadir fenicio, recogía la preocupación de los asistentes por la conservación y mantenimiento del patrimonio arqueológico $\mathrm{y}$, entre las demandas básicas del sector, reclamaba un epígrafe propio en la Clasificación Nacional de Actividades Económicas (CNAE) y los derechos laborales inherentes a la profesión, la inclusión de los profesionales de la arqueología en el ámbito de aplicación del Estatuto del Artista y de los Trabajadores de la Cultura, la reforma y racionalización de las legislaciones específicas, tanto a nivel estatal, como autonómico o el establecimiento de la colegiación obligatoria en tanto que el trabajo arqueológico se desarrolla íntegramente sobre bienes de titularidad pública, entre otras propuestas. Finalmente, se planteaba la creación de una Plataforma Estatal de Profesionales de la Arqueología para desarrollar las líneas de trabajo salidas de este primer encuentro.

A esta primera cita se dio continuidad con un segundo encuentro, la Reunión de Arqueología Profesional, organizada por el Colegio de Madrid y celebrada en la sede colegial en abril de 2019. Respecto al encuentro anterior de Cádiz, que había supuesto una ruptura definitiva con las ineficientes líneas de trabajo desarrolladas hasta entonces, la reunión de Madrid incorporó dos novedades que resultarían esenciales en el posterior proyecto "Innovando (desde) el Ecosistema Arqueológico Profesional": la superación del marco colegial que había guiado al encuentro en Cádiz seis meses antes, abriendo la participación a personas colegiadas, no colegiadas, pertenecientes a asociaciones profesionales, no adscritas de ninguna manera o estudiantes de los últimos años de grado, y el abandono del sistema habitual de mesas redondas o charlas magistrales con debate posterior para sustituirlo por una asamblea abierta facilitada por personas ajenas 
al mundo de la arqueología, en la que los temas de debate y discusión se decidían sobre la marcha al comienzo de cada sesión.

En este encuentro se incidió en el marcado carácter social que debe tener el trabajo arqueológico, para lo cual se proyectó, al inicio de las sesiones, el cortometraje "Una pregunta muy sencilla", elaborado a base de preguntas a ciudadanos que acababan de visitar el $\mathrm{Mu}-$ seo Arqueológico Nacional y a los que se les preguntaba qué era para ellos la arqueología, cómo veían la profesión arqueológica y qué echaban de menos en nuestra labor. El video ${ }^{2}$ sirvió para poner en situación a los asistentes sobre qué demandaba de la profesión arqueológica la sociedad a la que nuestro trabajo va dirigido.

Después de una primera toma de contacto, se establecieron cuatro líneas de trabajo que, en adelante, habrían de ser esenciales en todo el camino recorrido hasta el momento: condiciones laborales, aspectos legales, arqueología y sociedad y arqueología en común. Durante las sesiones, se plantearon diversos escenarios ideales de futuro (entre otros, "La sociedad demanda Arqueología porque desde la infancia tenemos acceso a esos saberes y aprendemos a valorarlos, gracias a la educación" o "La profesión de la arqueología está regulada y reconocida en sus diferentes ámbitos") y, por primera vez se definió el "Arqueoecosistema" como "Un marco de relación público-social en el que la Arqueología es garante de una relación virtuosa. Un marco en el que lo público asume obligaciones (deberes) y en el ámbito de lo social se ponen en valor derechos". Al final de la reunión, se establecieron grupos de trabajo que dieran continuidad a la labor realizada y, finalmente, se acordó retomar la conversación en un encuentro posterior.

En las semanas siguientes fue tomando cuerpo, en torno a esa idea del Arqueoecosistema, un proyecto que, mediante una serie de reuniones organizadas por colegios profesionales en diversos puntos del Estado, permitiera poner sobre la mesa los problemas que cada colectivo tiene en el ámbito donde desarrolla su trabajo, buscar entre todos soluciones comunes para esos problemas y, especialmente, ir formando un colectivo arqueológico que trascendiera los límites de la comunidad autónoma en la que cada cual ejerce principal-

"Una pregunta muy sencilla": https://www.youtube.com/ watch? $\mathrm{v}=\mathrm{qEaegTzWKE0}$ mente su trabajo: es decir, formar un grupo de profesionales cohesionado capaz de, innovando los métodos utilizados hasta ahora, consolidar la profesión arqueológica. Así nació el proyecto "Innovando (desde) el Ecosistema Arqueológico Profesional", coordinado por el Colegio de Profesionales de la Arqueología de Madrid y organizado también por sus homónimos de Cantabria y Granada - Almería - Jaén. Con el proyecto ya en marcha, la concesión de una ayuda a la Acción y Promoción Cultural por parte de la Dirección General de Industrias Culturales y Cooperación del Ministerio de Cultura y Deporte, en su convocatoria de 2019, facilitó la consecución de los objetivos del proyecto y la realización de las actividades que lo conformaban.

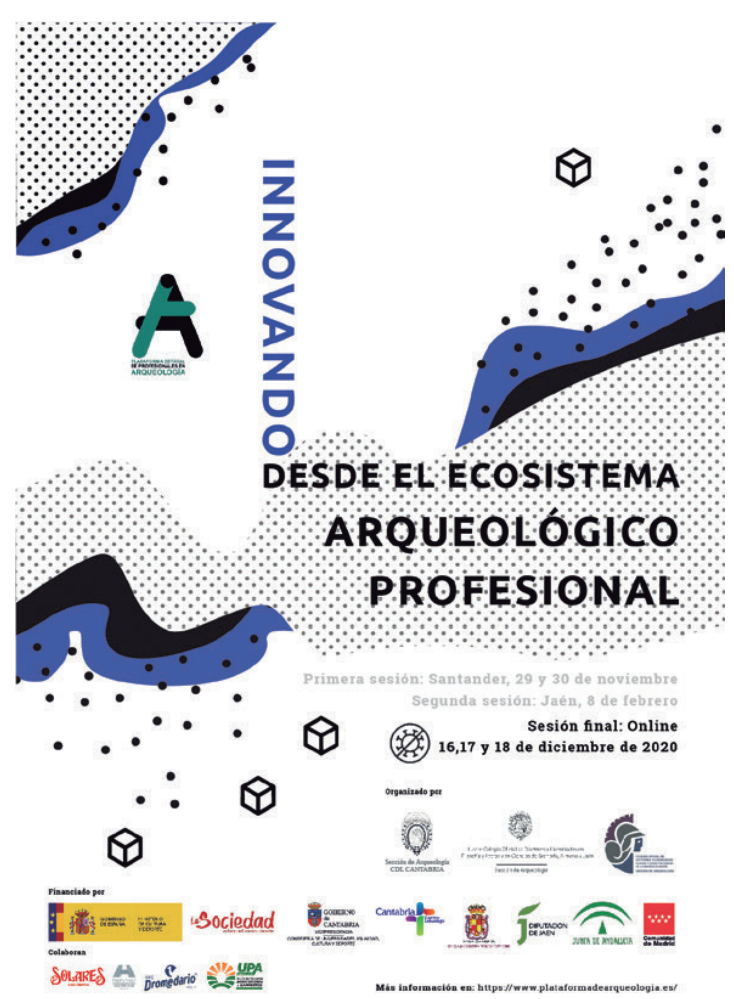

Figura 3. Cartel de la última sesión del proyecto, prevista para marzo de 2020 en Madrid y, finalmente, aplazada al mes de diciembre de ese mismo año (PEPA, 2020)

La primera de las reuniones programadas, organizada por el Colegio de Cantabria, tuvo lugar en Santander, los días 29 y 30 de noviembre de 2019. Después de un primer encuentro (o reencuentro) celebrado la tarde del día 29, tuvo lugar una sesión de trabajo en el denomi- 
nado Enclave Pronillo, el rehabilitado Palacio de Riva - Herrera, en Santander, para la que fue fundamental la cooperación de la Vicepresidencia y Consejería de Universidades, Igualdad, Cultura y Deporte, y de la Sociedad Regional de Educación, Cultura y Deporte. Se mantuvo el formato de espacio abierto utilizado ya en la reunión de Madrid y, después de unos primeros momentos dedicados a identificar a los participantes en la reunión y a facilitar la interacción entre ellos, se llevó a cabo una dinámica en la que se trabajó por grupos en torno a preguntas relacionadas con la imagen que se da de nuestra profesión y perspectivas de futuro. De esta primera dinámica surgió una definición consensuada según la cual los presentes aspiraban a que la arqueología profesional fuera "un colectivo profesional diverso, reconocido y prestigiado por la sociedad e interconectado con ella".

La segunda parte de la jornada estuvo dedicada a debatir sobre la necesidad o no de crear una nueva estructura bajo cuyo paraguas pudiese desarrollarse la arqueología profesional, y se consideró esencial elaborar un censo real que incluya a todas las personas que en el Estado se dedican a la arqueología profesional, así como seguir trabajando tanto dentro de las estructuras ya existentes, como en la creación de otras que resulten más eficaces. Como resumen, cabe decir que en la reunión de Santander se establecieron dos líneas de trabajo para avanzar en el diseño del plan de innovación que consolide el futuro del sector. Por un lado, los arqueólogos se comprometieron a seguir dando pasos a través de las Secciones de Arqueología de los Colegios de Doctores y Licenciados en Filosofía y Letras y en Ciencias que existen en algunas comunidades autónomas, dentro del marco del Consejo General, organismo que representa a los profesionales a nivel nacional. Al mismo tiempo, se formaron grupos de trabajo para buscar sinergias con otros sectores, mejorar la conexión con la sociedad y diseñar un plan de comunicación que favorezca la visibilidad del colectivo, entre otras cosas. El acuerdo final fue conocido entre los profesionales del sector, a partir de entonces, como el Pacto de Santander.

Ante la imposibilidad de conocer de antemano las circunstancias que condicionarían la fase final del proyecto a partir de marzo de 2020, se convocó la siguiente reunión, en Jaén, en el mes de febrero de ese año. La idea inicial era que el encuentro de Santander hubiera servido para reunir a los profesionales de la mitad norte de la península, mientras que el de Jaén facilitaría la cita de quienes desarrollan su actividad en la mitad meridional. En ese contexto, el encuentro final, en Madrid, sería la gran reunión de todas las personas que se dedican a la arqueología profesional en España.

Con ese horizonte, la reunión de Jaén tuvo lugar los días 7 y 8 de febrero de 2020, con la organización del Colegio de Granada, Almería y Jaén (conocido frecuentemente por su acrónimo CODOLI). En líneas generales, las sesiones de trabajo, desarrolladas en el Archi-

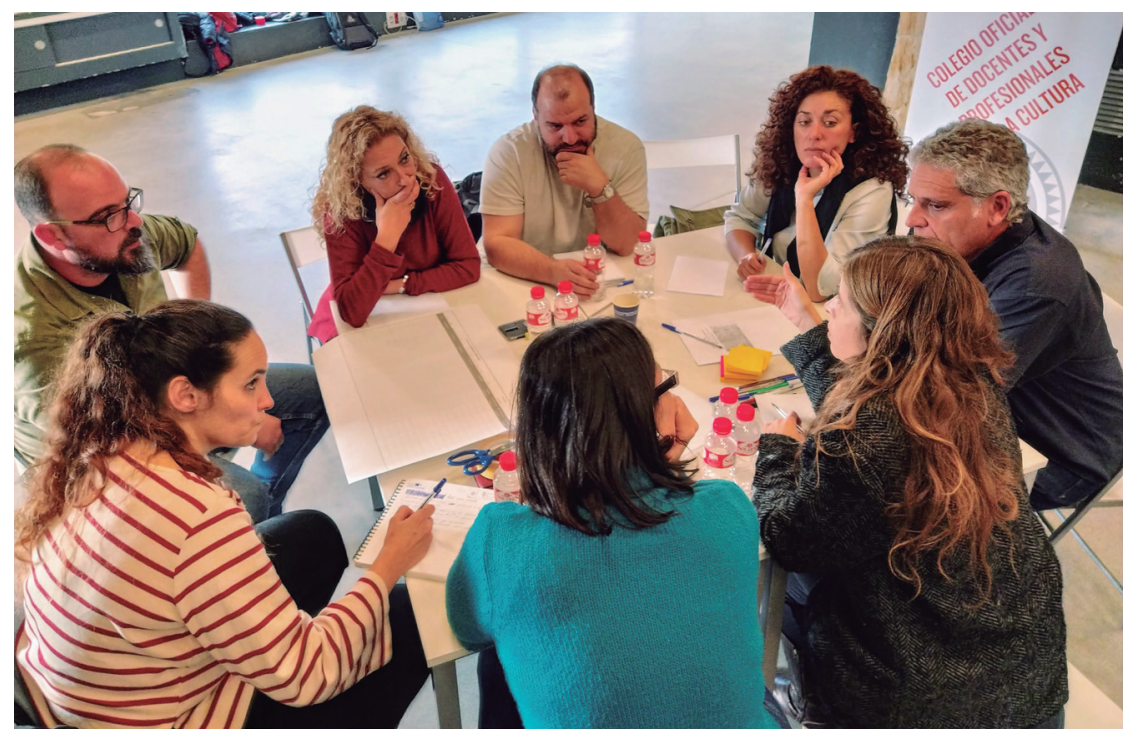

Figura 4. Santander, un momento de la sesión matinal (Firenze Comunicación, 2019). 


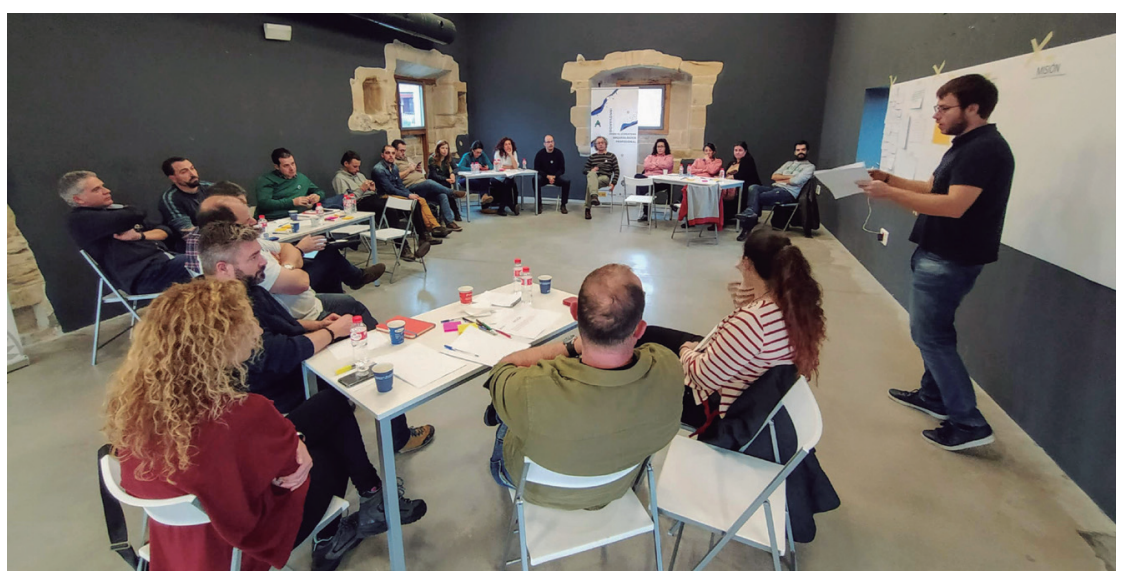

Figura 5. Santander, espacio abierto en la sesión de tarde (Firenze Comunicación, 2019).

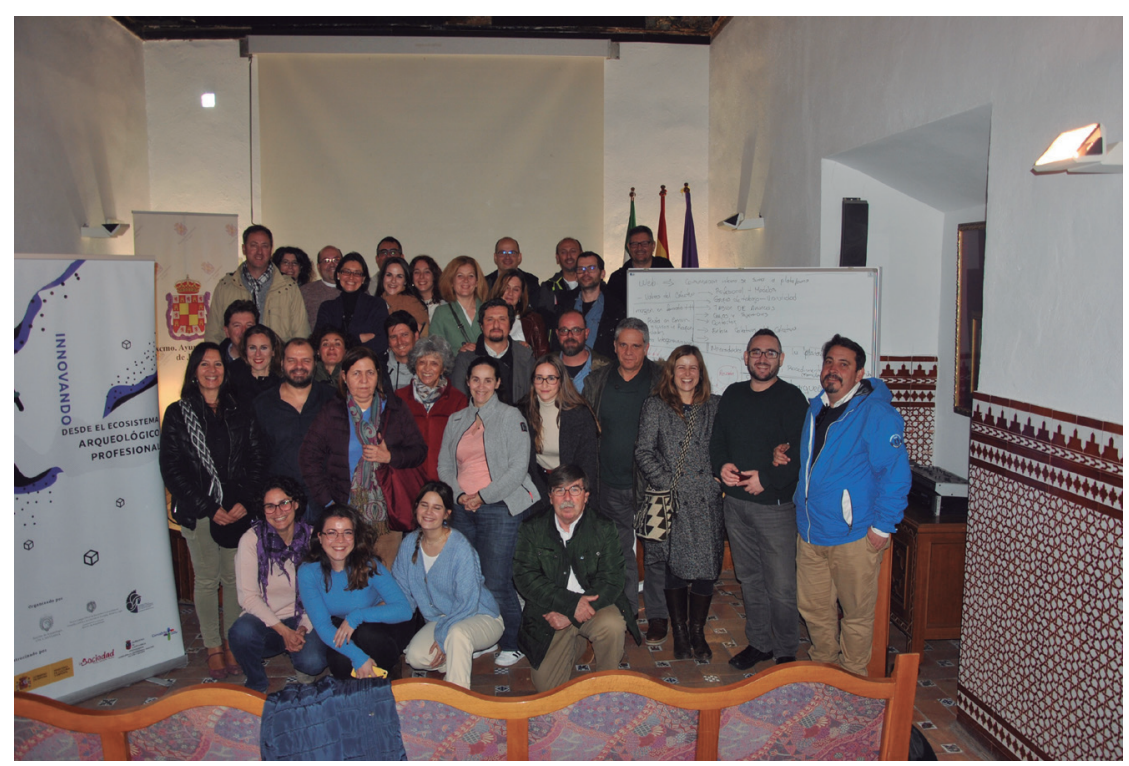

Figura 6. Jaén, los participantes al terminar las sesiones (Laboratorio de Cuidados Urbanos, 2020).

vo Histórico Provincial de Jaén y en el Salón Mudéjar del Ayuntamiento, dieron un paso adelante con respecto a lo tratado en Santander y concluyeron, en una declaración final conocida como "Acuerdo de Jaén", que "Las personas presentes en el Archivo histórico de Jaén el ocho de febrero de dos mil veinte, sintiéndose en continuidad con la conversación abierta en Cádiz en 2018 y abundada en Madrid y en Santander en 2019 se comprometen a apoyar tanto 1) la transformación de la Comisión de Arqueología del Consejo General dotándola de un reglamento elaborado democráticamente que la oriente a la defensa de la profesión como 2) el impulso del ecosistema alrededor de la arqueología lo más amplio posible a través de una plataforma"

La siguiente reunión, que debía ser la última, se programó para el último fin de semana de marzo de 2020. Sin embargo, la situación derivada de la crisis sanitaria causada por la COVID - 19, con la suspensión sine die de todas las actividades que no resultasen esenciales, obligó a aplazar esa cita que, organizada por el Colegio de Madrid, debía haberse celebrado, además de en la sede del propio Colegio, en el Museo Arqueológico Regional y en el Archivo Regional de la Comunidad de Madrid. En un primer momento, confiando en la posibilidad de que la situación permitiese 
realizar un encuentro presencial, valorando que el proyecto se sustentaba en el encuentro entre profesionales, se fijó una fecha a finales de septiembre; la realidad, una vez más, se impuso con terquedad y obligó a aplazar el encuentro hasta apurar el plazo concedido por el Ministerio de Cultura para el desarrollo del proyecto. De este modo, la sesión final de "Innovando (desde) el Ecosistema Arqueológico Profesional" se llevó a cabo entre los días 16 y 18 de diciembre, las dos primeras reuniones en la sede del Colegio de Madrid, y la última, en el Museo Arqueológico Regional, en Alcalá de Henares.

La primera sesión, titulada "Nuevos profesionales", centrada en la presentación de nuevos enfoques de la arqueología y dirigida especialmente a estudiantes y a recién graduados, contó con la participación de Juanjo Pulido y Sabah Walid, de la empresa Underground: arqueología, patrimonio y gente, que expusieron sus líneas de trabajo, muy cercanas siempre a la sociedad y, en particular, a la ciudadanía del entorno inmediato donde se desarrolla cada actuación: procesos comunitarios y patrimonio, equipos híbridos en los proyectos de arqueología, metodologías procesuales y participativas para el acercamiento de la arqueología a la gente, relacionando las excavaciones con el paisaje de vida y con las comunidades para entender el hilo de la historia. A ellos se debe, también, la iniciativa de organizar el Congreso Internacional de Socialización del Patrimonio en el Medio Rural, conocido como SoPa. Esa reunión, en fin, se enmarca dentro del conjunto de iniciativas (como el TAG Ibérico, celebrado en Carmona, o las diferentes sesiones de Arqueonet, en Madrid), que estaban fraguando el caldo de cultivo que debía convulsionar la profesión arqueológica.

La siguiente intervención correspondió a Miguel Fernández Díaz, arqueólogo especializado en la virtualización y digitalización del patrimonio, trabajo que desarrolla en la empresa Virtua Nostrum. Expuso su trayectoria profesional y las posibilidades que abren las nuevas tecnologías en la arqueología actual. Como ejemplo de nuevas posibilidades de asociacionismo explicó su experiencia en ADARQ, la Asociación de Dibujantes de Arqueología.

La última intervención de esta primera sesión estuvo a cargo de Pablo González Zambrano, en representación del Sindicato Andaluz de Trabajadores, Sección Universidad de Granada. En su ponencia, que aportó la visión de la nueva generación de arqueólogos, denunció el intrusismo laboral en el ámbito de la arqueología, puesto que "cualquiera que justifique horas de trabajo en campo puede ejercer como arqueólogo". Cualquier persona

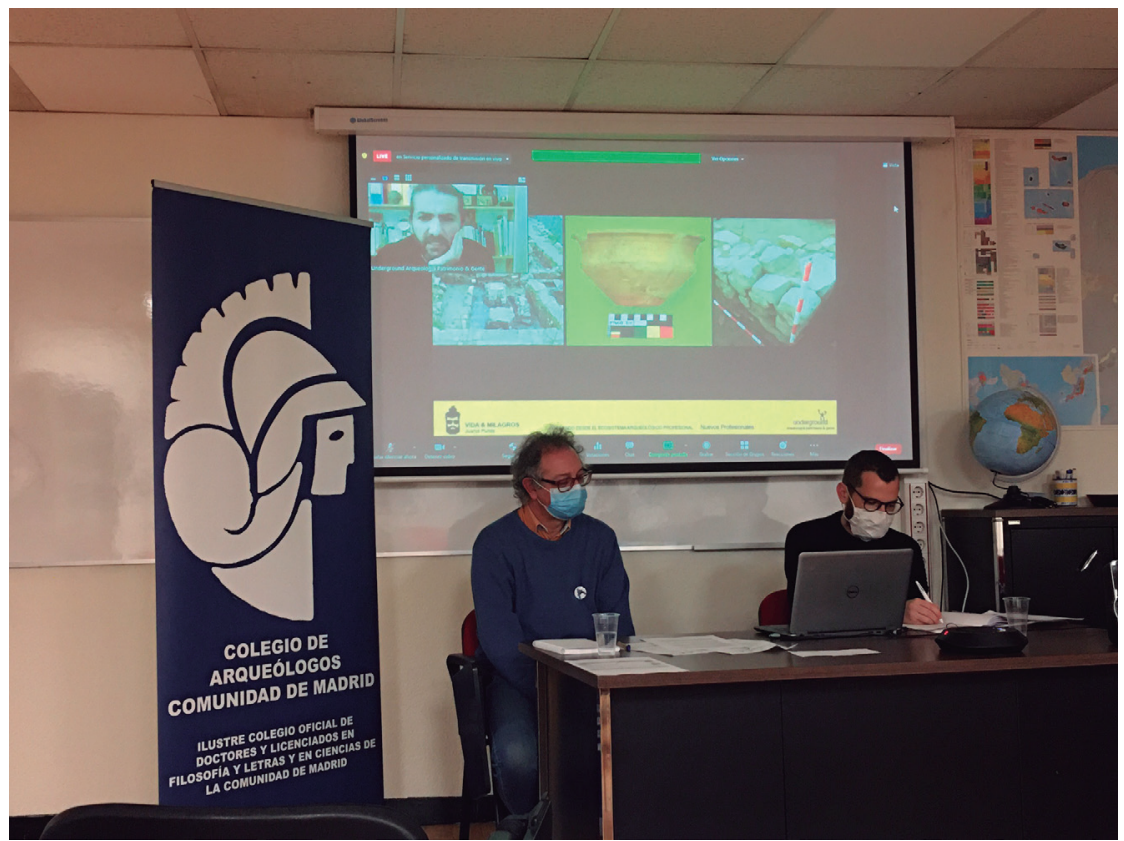

Figura 7. Madrid, una de las sesiones online (Laboratorio de Cuidados Urbanos, 2020). 
con título en historia, historia del arte, antropología, humanidades, etc. con experiencia en campo puede ejercer como arqueólogo. En este sentido, el intrusismo laboral es elevado y la pérdida de calidad de los trabajos también. Por tanto, no se privilegia en el ámbito del patrimonio al graduado en arqueología. "Somos una clase obrera desprovista de derechos", concluyó.

La segunda sesión, desarrollada el 17 de diciembre bajo el título "cultura organizacional", incidió en las distintas formas de asociacionismo que han elegido profesionales tanto de la arqueología, como de disciplinas cercanas. Para ello, se contó con la participación de Isidre Pastor, en representación de la Associació d'Arqueolègs de Catalunya (AD'AC), que expuso el recorrido de esta asociación profesional desde sus comienzos, en los años 90 del siglo XX, hasta convertirse, en la actualidad, en el marco de referencia para los profesionales que ejercen la arqueología en Catalunya.

La siguiente intervención correspondió a Cristina San Martín, en representación de la Asociación Profesional de Conservadores Restauradores de España (ACRE), que planteó en su exposición cómo en la restauración de bienes culturales se dan situaciones muy similares a las existentes en la arqueología profesional (desregulación de una profesión no reconocida, intrusismo profesional, destrucción del patrimonio por malas prácticas no controladas...) que dieron lugar al nacimiento de esta Asociación, que se ha consolidado, en sus apenas 10 años de vida, como un interlocutor válido con todos los agentes implicados en la gestión del patrimonio cultural.

Para la última exposición de la tarde se invitó a Mauge Cañada en representación de IIFACE, Instituto de Facilitación y Cambio, quien, frente a los planteamientos eminentemente prácticos de los representantes de AD'AC y ACRE, se centró en el marco teórico en el que nuevas formas de asociacionismo profesional son posibles.

La jornada que cerró el proyecto se desarrolló, a lo largo del día 18 de diciembre, en el Museo Arqueológico Regional. En la sesión de mañana, dedicada a "La arqueología en las instituciones", se contó con Benito Burgos, como representante del Ministerio de Cultura y Deporte, donde coordina el programa "Cultura y Ciudadanía". En su intervención insistió en la cultura y el patrimonio como hechos sociales en los que es imprescindible abrir espacios para la participación de la ciudadanía. Participó también Isabel Baquedano, en representación de la Comunidad de Madrid, donde es Jefa del Área de Protección del Patrimonio Arqueológico, quien, además de explicar pormenores de la gestión del patrimonio arqueológico desde la Administración, puso el acento sobre la invisibilización de la arqueología profesional en los medios de comunicación y la distancia que existe entre el trabajo de los profesionales y la sociedad.

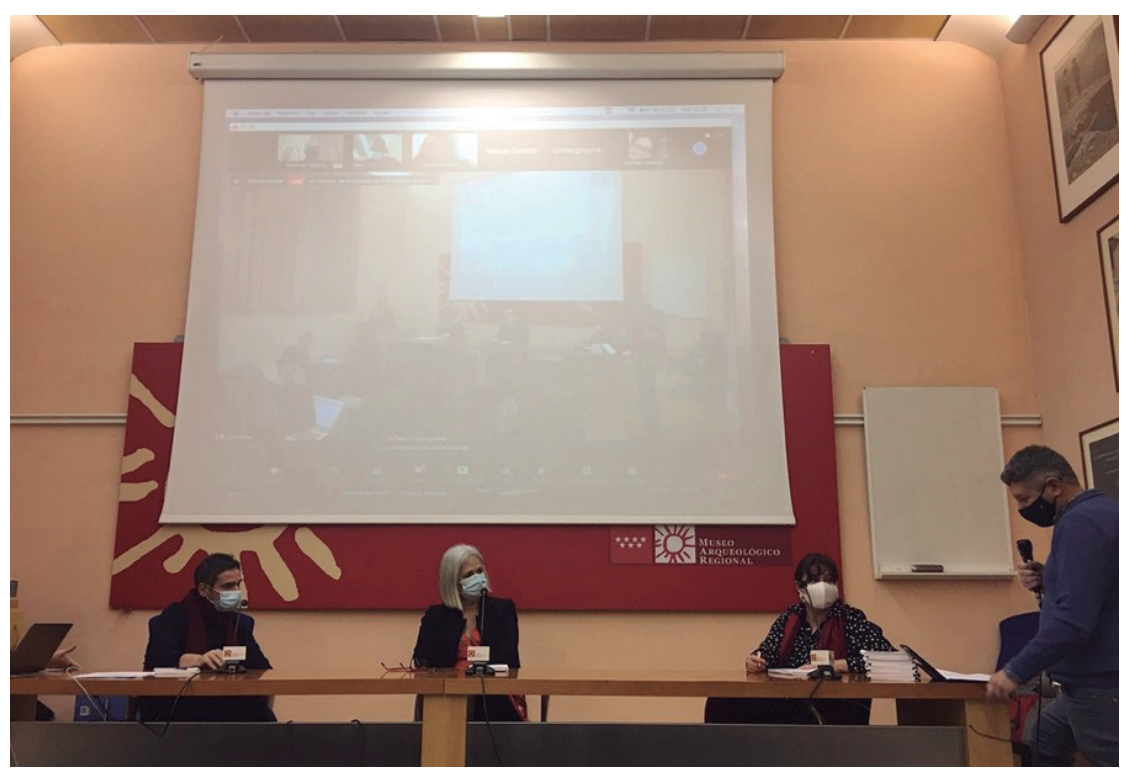

Figura 8. Madrid, sesión final en el Museo Arqueológico Regional (Laboratorio de Cuidados Urbanos, 2020). 
La participación en la mesa redonda la cerró Alicia Castillo, presidenta del Comité Nacional de España de ICOMOS (el Comité Internacional de Monumentos y Sitios) y profesora de la Universidad Complutense. Insistió en la incapacidad de quienes ejercen profesionalmente la arqueología para transmitir quiénes son, en qué consiste o para qué sirve su trabajo y, en ese sentido, apuntó la idea de que la arqueología requiere ser pensada de forma compleja, con la ciudadanía, con otras profesiones y disciplinas, incluso también con la ecología.

Como moderador de la sesión ejerció Ángel Astorqui, presidente de la Plataforma de Empresas Culturales de Cantabria quien, desde esa perspectiva, aportó también su visión de las enormes posibilidades que ofrece el asociacionismo en el sector cultural para la consecución de objetivos comunes.

La sesión de tarde recuperó la metodología de espacio abierto usada en las reuniones anteriores, si bien limitada por las circunstancias de que el debate se desarrolló por vía telemática. Se expuso el punto en el que estaba la creación de nuevas estructuras para la cohesión del colectivo arqueológico profesional, se emplazó a los asistentes a la primera reunión de trabajo de la ya constituida "Plataforma Estatal de Arqueología Profesional", de la que saldrían los estatutos, y se puso punto final al proyecto "Innovando (desde) el Ecosistema Arqueológico Profesional".

\section{Caminando hacia el futuro: la consolidación del colectivo}

Tras la finalización del proyecto "Innovando (desde) el Ecosistema Arqueológico Profesional" se ha constituido un nuevo sistema de relaciones entre quienes integran la arqueología profesional en España, mucho más sólido que el existente con anterioridad. El proyecto ha supuesto dar pasos adelante con respecto a lo que el colectivo arqueológico ya había ido realizando en reuniones anteriores (Cádiz 2018, Madrid 2019), y permite superar marcos organizativos restrictivos cuyo funcionamiento era ajeno a la realidad de la profesión arqueológica.

En la actualidad, tras el impulso dado por el proyecto financiado por el Ministerio de Cultura en 2019 y 2020, la arqueología profesional en España se agrupa en torno a la Plataforma
Estatal de Profesionales de la Arqueología, que ha redactado ya sus estatutos y que sigue celebrando, con regularidad, reuniones que permitan definir su modo de funcionamiento. Desde esta recién creada Plataforma, se han realizado ya varias acciones para reivindicar la defensa de la profesión y la del patrimonio cultural que pertenece, íntegramente, a la ciudadanía de la que los profesionales de la arqueología también forman parte.

En ese sentido, se ha hecho un gran esfuerzo en comunicación, para transmitir de la mejor manera posible los resultados alcanzados con el proyecto. Y se demanda, además, de la Administración que la reforma de la vigente Ley del Patrimonio Histórico Español, de la que emanarán, como ya sucedió, las legislaciones de ámbito local, tenga en cuenta cuanto aquí se ha dicho acerca de la financiación de las actividades arqueológicas.

De esta manera, el resultado más visible del proyecto financiado con la ayuda de la Dirección General de Industrias Culturales y Cooperación del Ministerio de Cultura y Deporte ha sido la constitución de la "Plataforma de Profesionales de la Arqueología" (https://plataformadearqueologia.es/), de la que forman parte profesionales de todo el Estado que aportan sus diferentes experiencias y sus preocupaciones y problemas para buscar una solución común y consolidar la Arqueología como una profesión. Puede decirse que la formación de esta plataforma es el intento más serio realizado hasta el momento en el Estado español para involucrar a todas las personas que ejercen la arqueología profesional en la consecución de un objetivo común que es compartir intereses, problemas y soluciones.

Ningún proyecto de consolidación de la disciplina arqueológica debería, en fin, olvidar que "La ciudadanía es el fin último de los procesos culturales, y son varias las formas en las que se relaciona con esos procesos: bien como consumidora de productos culturales, bien implicándose de forma activa tanto en el diseño como en la toma de decisiones sobre la programación cultural de su municipio, o bien formando parte de la generación del contenido cultural y del conocimiento sobre el patrimonio de su territorio cercano" (Pulido 2015: 66). Sin la ciudadanía, en fin, nuestro trabajo resulta por completo incomprensible. 


\section{Bibliografía}

Almansa, J. (ed.) (2011): El futuro de la arqueología en España. JAS Arqueología, Madrid

Almansa, J. (ed.) (2013): Arqueología pública en España. JAS Arqueología, Madrid

Almansa, J. (2018): Arqueología y sociedad: interacción y acción desde la teoría crítica. Tesis doctoral no publicada. Universidad Complutense de Madrid.

Almansa, J. (2021): Del Mediterráneo a Madrid. \#pubarchMED y viejas-nuevas perspectivas en la gestión del patrimonio arqueológico. Reunión de Arqueología Madrileña 2019. Ilustre Colegio Oficial de Doctores y Licenciados en Filosofía y Letras y en Ciencias de la Comunidad de Madrid, Madrid: 283-290

Aparicio, P. (2018): Mercenarios en la selva del precariado: construyendo la arqueología virtual como profesión. Actas del Congreso Nacional de Arqueología Profesional, (J.I. Lorenzo, ed.), Colegio Oficial de Doctores y Licenciados en Filosofía y Letras y en Ciencias de Aragón, Zaragoza: 19-26

Astorqui, A. y Díaz, Y. (2018): Del modelo tradicional a la arqueología pública: El caso Cooltoure, egoístamente, por y para las personas. Actas del Congreso Nacional de Arqueología Profesional, (J.I. Lorenzo, ed.), Colegio Oficial de Doctores y Licenciados en Filosofía y Letras y en Ciencias de Aragón, Zaragoza: 417-421

Baena, J. (2007): Profesionales de la arqueología: caminos para un mismo fin. Actas de las Segundas Jornadas de Patrimonio Arqueológico en la Comunidad de Madrid, Comunidad de Madrid Consejería de Cultura y Turismo, Madrid: 117-125

Barrachina, A. y Selma, S. (2014): Arqueología de los servicios públicos versus arqueología privada. Reflexión sobre sus puntos de encuentro y sus desavenencias. La Linde. Revista Digital de Arqueología Profesional, 2: 92-99

Castillo, A. (2004): La gestión del Patrimonio Arqueológico y el urbanismo en la Comunidad de Madrid. Complutum, 15: 99-144

Corpas, N.; Sánchez García, D. y Castillo, A. (2019): ALCALÁ PAST COMUN. Construyendo un pasado común en Alcalá de Henares. Reunión de Arqueología Madrileña 2018, Ilustre Colegio Oficial de Doctores y Licenciados en Filosofía y Letras y en Ciencias de la Comunidad de Madrid, Madrid: 111-120.

Fernández Ugalde, A.; Marín, F.J.; Mena, P. y Serrano, E. (1998): Las murallas de Madrid. Arqueología medieval urbana, Dirección General de Patrimonio Cultural, Madrid

Gibaja, J.; Higueras, S. y Torres, J.R. (2018): Implicación de la ciudadanía en actividades divulgativas: "Barcelona, una historia de hace 6.000 años". Actas del Congreso Nacional de Arqueología Profesional, (J.I. Lorenzo, ed.), Colegio Oficial de Doctores y Licenciados en Filosofía y Letras y en Ciencias de Aragón, Zaragoza: 375-382

Gómez Hernanz, J. (2007): La Arqueología en el ámbito profesional: una síntesis de la sesión. Actas de las Segundas Jornadas de Patrimonio Arqueológico en la Comunidad de Madrid, Comunidad de Madrid Consejería de Cultura y Turismo, Madrid: 137

López Martínez, L. y Martín Alonso, J. (2018): ¿Han ganado ellas? Arqueólogas vs. Constructoras Actas del Congreso Nacional de Arqueología Profesional, (J.I. Lorenzo, ed.), Colegio Oficial de Doctores y Licenciados en Filosofía y Letras y en Ciencias de Aragón, Zaragoza: 225-233

Lorenzo, J.I. (2018): La arqueología profesional en España: 27 años construyendo la profesión desde los Colegios Oficiales de Doctores y Licenciados en Filosofía y Letras y en Ciencias. Actas del Congreso Nacional de Arqueología Profesional, (J.I. Lorenzo, ed.), Colegio Oficial de Doctores y Licenciados en Filosofía y Letras y en Ciencias de Aragón, Zaragoza: 9-11

Mancilla, M.I.; García González, D.; Moreno, A. y Sánchez Gómez, P. (2018): Arqueología y sociedad. "El Patrimonio Arqueológico: de las trincheras a la sociedad. La Granada invisible". Actas del Congreso Nacional de Arqueología Profesional, (J.I. Lorenzo, ed.), Colegio Oficial de Doctores y Licenciados en Filosofía y Letras y en Ciencias de Aragón, Zaragoza: 395-404

Mena, P. (1991): Arqueología urbana en el término municipal de Madrid (1985-1990). Arqueología, Paleontología y Etnografia, 1: 201-216

Moya, P.R. (2010): Grandezas y miserias de la arqueología de empresa en la España del siglo XXI. Complutum, 21: 9-26

Palomero, G. (2019): Estudiando arqueología: la formación como mercancía, la respuesta estudiantil y otras locuras del sistema neoliberal. Reunión de Arqueología Madrileña 2018, Ilustre Colegio Oficial 
de Doctores y Licenciados en Filosofía y Letras y en Ciencias de la Comunidad de Madrid, Madrid: 322-332

Parga - Dans, E. (2014): La arqueología profesional en España analizada desde la sociología (entrevista). La Linde. Revista Digital de Arqueología Profesional, 3: 12-18

Parga - Dans, E.; Alonso - González, P. y Otero, R. (2020): The social value of heritage: Balancing the promotion-preservation relationship in the Altamira World Heritage Site, Spain. Journal of destination Management and Marketing, 18.

Pastor, A. y Ruiz Martínez, A. (2016): Nuevas metodologías para una comprensión de las interacciones entre el público y el patrimonio arqueológico urbano. Arqueología y Comunidad. El valor del Patrimonio Arqueológico en el siglo XXI, JAS Arqueología, Madrid: 91-112

Pastor, A. (2019): Conservación arqueológica social. Etnografias patrimoniales en el Barri Gòtic de Barcelona. Tesis doctoral no publicada. Universitat de Barcelona

Polo, J. (2007): La Arqueología en el ámbito profesional de la Comunidad de Madrid. La mayoría de edad. Actas de las Segundas Jornadas de Patrimonio Arqueológico en la Comunidad de Madrid, Comunidad de Madrid Consejería de Cultura y Turismo, Madrid: 131-135

Pulido, J. (2015): La socialización del patrimonio: aclarando conceptos, centrifugando ideas. La Linde. Revista Digital de Arqueología Profesional, 4: 65-82

Ruiz Martínez, A. y Pastor Pérez, A. (2015): Hacia una arqueología social en el centro de Barcelona. Aproximación metodológica para el estudio del uso del patrimonio urbano. La Linde. Revista Digital de Arqueología Profesional, 5: 140-192

Ruiz Taboada, A. (2014): La arqueología se ha convertido en un mero trámite incómodo en los proyectos de rehabilitación urbana. Revista PH, 86: 19-23

Vaquerizo, D. (2015): Arqueología para un futuro incierto: la profesión de arqueólogo tras la crisis devastadora del "pelotazo". Pyrenae, 46 (2): 89-120

Vigil - Escalera, A. (2011): El pequeño mundo en ruinas de la arqueología contractual española. Arkeogazte, 1: $17-20$

Vizcaíno, A. (2015): Dilemas, reflexiones y posibilidades de una investigación arqueológica que se pretende socialmente comprometida. La Linde. Revista Digital de Arqueología Profesional, 5: 193-214 
